\title{
Evaluation of an Expired Nontoxic Amlodipine Besylate Drug as a Corrosion Inhibitor for Low-Carbon Steel in Hydrochloric Acid Solutions
}

\author{
A. S. Fouda ${ }^{1}$ - W. M. Mahmoud ${ }^{1}$ - H. A. Abdul Mageed ${ }^{1}$
}

Received: 25 November 2015/Revised: 21 February 2016/Accepted: 3 March 2016/Published online: 22 March 2016

(C) Springer International Publishing Switzerland 2016

\begin{abstract}
The corrosion inhibition of Amlodipine Besylate drug as a corrosion control of low-carbon steel in $1 \mathrm{M}$ $\mathrm{HCl}$ solution was investigated by electrochemical and chemical techniques. The inhibition efficiency (\%IE) was found to increase with an increase in drug concentrations but deceases with increasing the temperature. Results showed that this drug acts as a mixed-type inhibitor. It was found that this drug acts via adsorption on the low-carbon steel surface and the adsorption obeys Langmuir adsorption isotherm. Quantum structure-activity relationships have been studied. The surface morphology of low-carbon steel samples was investigated.
\end{abstract}

Keywords Corrosion inhibition $\cdot$ Low-carbon steel $\cdot \mathrm{HCl}$ - Amlodipine Besylate drug

\section{Introduction}

Corrosion is a costly and severe materials science problem. The corrosion of low-carbon steel is the most common form of corrosion, especially in acid solution. It has received a considerable attention as a result of its industrial importance, for example in the chemical cleaning and processing, oil well acidizing, and petrochemical industry. Low-carbon steel which is subjected to painting, electroplating, phosphate coating, and cold rolling must have a clean surface free from oxide scale. To remove unwanted scale, the low-carbon steel is immersed in an acid solution, namely in an acid pickling

A. S. Fouda

asfouda@hotmail.com

1 Chemistry Department, Faculty of Science, Mansoura University, Mansoura 35516, Egypt bath. Hydrochloric acid solutions are by far the most widely used in pickling bath of low-carbon steel. Because of the general aggressiveness of acid solutions, organic inhibitors are commonly used to reduce the corrosion attack on metallic materials. Majority of the well-known inhibitors for the corrosion of steel in acidic medium are organic compounds containing nitrogen, sulfur, oxygen atoms, or N-hetero cyclic compounds with polar groups. A large number of scientific studies have been devoted to the subject of corrosion inhibitors for low-carbon steel in acidic media [1-12]. Most of the commercial inhibitors are toxic in nature; therefore, replacement by environmentally benign inhibitors is necessary. Few nontoxic compounds have been investigated as corrosion inhibitors by some researchers [13, 14]. The use of drugs offers interesting possibilities for corrosion inhibition due to the presence of heteroatoms like nitrogen, sulfur, and oxygen in their structure, and they are of particular interest because of their safe use, high solubility in water, and high molecular size. Most of the drugs are more expensive than organic inhibitors, and therefore expired drugs can be used as corrosion inhibitors. The use of expired drugs can solve two major environmental and economic problems: limitation of environmental pollution with pharmaceutically active compounds and reduction of the disposal costs of expired drugs. It is reported that the active constituent of the drug degrades only infinitesimally. More than $90 \%$ of the drugs maintained stability long time after the expiry dates [15]. Nicolae has tested two expired drugs carbamazepine and paracetamol as corrosion inhibitors on carbon steel corrosion in $\mathrm{H}_{2} \mathrm{SO}_{4}$ and $\mathrm{CH}_{3} \mathrm{COOH}$ and obtained 95 and $85 \%$ efficiency, respectively [16]. Some of the azosulfa and antimalarial drugs have been reported as good corrosion inhibitors [17-19]. Also, the use of piperazine [20], septazole [21], and cefixime [22] drugs as corrosion inhibitors has been reported. 
In the present work, expired Amlodipine Besylate drug has been investigated as a corrosion inhibitor for low-carbon steel in hydrochloric acid using different techniques. Amlodipine Besylate contains $\mathrm{N}$ atoms, $\mathrm{O}$ atoms, $-\mathrm{CH}_{3}$, $\mathrm{NH}_{2}$ groups, and $\pi$-bond in its structure and has higher molecular size, which are regarded as important factors for good inhibitor performance. Amlodipine Besylate drug is used primarily for lowering blood pressure, to lower the risk of stroke and heart attack, and to prevent chest pains.

\section{Experimental Methods}

\subsection{Materials and Solutions}

Amlodipine Besylate (99.8 \% anhydrous) was supplied by Egyptian international pharmaceutical industries company (EIPICO), 10th Ramadan, Egypt. The drug was used without further purification. The test material used was low-carbon steel sample with the following composition (wt \%): $0.14 \mathrm{C}, 0.6 \mathrm{Mn}, 0.05 \mathrm{~S}, 0.04 \mathrm{P}, 0.1 \mathrm{Si}$, and balance Fe. Test materials were abraded with different emery papers up to 1200 grade, cleaned with acetone, washed with double-distilled water, and properly dried prior to exposure. Analar grade $\mathrm{HCl}$ and double-distilled water were used to prepare all solutions.<smiles>CCOC(=O)C1=C(COCCN)NC(C)=C(C(=O)OC)C1c1ccccc1Cl</smiles>

(RS)-3-ethyl 5-methyl 2-[(2-aminoethoxy) methyl]-4-(2chlorophenyl)-6-methyl-1, 4-dihydropyridine-3, 5dicarboxylate

Molecular formula $\mathrm{C}_{20} \mathrm{H}_{25} \mathrm{Cl} \mathrm{N}_{2} \mathrm{O}_{5}$, Molecular weight $=$ 408.9

\subsection{Weight Loss Tests}

Low-carbon steel sheets of $20 \times 20 \times 2 \mathrm{~mm}$ were abraded with different grades of emery paper and then washed with double distilled water and acetone. After weighing accurately, the specimens were immersed in $100 \mathrm{ml} \mathrm{HCl}$ solution with and without addition of different concentrations of the drug. After $3 \mathrm{~h}$, the specimens were taken out, washed, dried, and weighed accurately. The average weight loss of the three parallel low-carbon steel sheets could be obtained at required temperature. The inhibition efficiency (IE) and the degree of surface coverage $(\theta)$ of the investigated drug on the corrosion of low-carbon steel were calculated as follows [23]:

$\% \mathrm{IE}=\theta \times 100=\left[1-\left(W / W^{\circ}\right)\right] \times 100$,

where $W^{\circ}$ and $W$ are the values of the average weight losses without and with the addition of the inhibitor, respectively.

\subsection{Electrochemical Measurements}

\subsubsection{Potentiodynamic Polarization Measurements}

Polarization experiments were carried out in a conventional three-electrode cell with platinum gauze as the auxiliary electrode and a saturated calomel electrode (SCE) coupled to a fine Luggin capillary as the reference electrode. The working electrode was in the form of a square cut from low-carbon steel sheet of equal composition embedded in epoxy resin of polytetrafluoroethylene so that the flat surface area was $1 \mathrm{~cm}^{2}$. Prior to each measurement, the electrode surface was pretreated in the same manner as the weight loss experiments. Before measurements, the electrode was immersed in solution for 30 min until a steady state was reached. The potential was started from -600 to $+400 \mathrm{mV}$ versus open circuit potential $\left(E_{\text {ocp }}\right)$. All experiments were carried out in freshly prepared solutions at $30{ }^{\circ} \mathrm{C}$ and were always repeated at least three times to check the reproducibility. Then $i_{\text {corr }}$ was used for the calculation of inhibition efficiency and surface coverage $(\theta)$ as follows:

$\% \mathrm{IE}=\theta \times 100=\left(1-\left(i_{\text {corr }} / i_{\text {corr }}^{\circ}\right)\right) \times 100$,

where $i^{\circ}{ }_{\text {corr }}$ and $i_{\text {corr }}$ are the corrosion current densities in the absence and presence of inhibitor, respectively.

\subsubsection{Electrochemical Impedance Spectroscopy (EIS) Tests}

Impedance measurements were carried out using AC signals of $5 \mathrm{mV}$ peak-to-peak amplitude at the open circuit potential in the frequency range of $100 \mathrm{kHz}$ to $0.1 \mathrm{~Hz}$. All impedance data were fitted to appropriate equivalent circuit.

\subsubsection{Electrochemical Frequency Modulation (EFM) Tests}

EFM experiments were performed with applying potential perturbation signal with amplitude $10 \mathrm{mV}$ with two sine waves of 2 and $5 \mathrm{~Hz}$. The choice for the frequencies of 2 and $5 \mathrm{~Hz}$ was based on three arguments [24,25]. The larger peaks were used to calculate the corrosion current density $\left(i_{\text {corr }}\right)$, the Tafel slopes $\left(\beta_{\mathrm{c}}\right.$ and $\left.\beta_{\mathrm{a}}\right)$, and the causality factors CF-2 and CF-3 [26]. The ${ }^{\circ}$ electrode potential was allowed to 
stabilize for $30 \mathrm{~min}$ before starting the measurements. All the experiments were conducted at $30^{\circ} \mathrm{C}$. Measurements were performed using Gamry Instrument Potentiostat/Galvanostat/ZRA (PCI4-G750). Gamry applications include DC 105 software for DC corrosion measurements, EIS 300 software for electrochemical impedance spectroscopy measurements, and EFM 140 for electrochemical frequency modulation measurements along with a computer for collecting data. Echem analyst $\mathrm{v} 6.03$ software was used for plotting, graphing, and fitting data.

\subsection{Quantum Calculations}

The molecular structure of the investigated compound was optimized initially with PM3 semi-empirical method so as to speed up the calculation. All the quantum chemical calculations were performed with Material studio V. 6.0.

\subsection{Scanning Electron Microscopy (SEM) Measurements}

The electrode surface of low-carbon steel was examined by scanning electron microscope-type JOEL 840, Japan, before and after immersion in $1 \mathrm{M} \mathrm{HCl}$ test solution in the absence and presence of the optimum concentrations of the investigated inhibitors at $25^{\circ} \mathrm{C}$, for an immersion time of 1 day. The specimens were washed gently with distilled water, then dried carefully, and examined without any further treatments.

\section{Results and Discussion}

\subsection{Chemical Method (Weight Loss Tests)}

Weight loss of low-carbon steel was determined, at various time intervals, in the absence and presence of different concentrations of Amlodipine Besylate. The obtained weight loss-time curves are represented in Fig. 1. The inhibition efficiency of corrosion was found to be dependent on the inhibitor concentration. The curves obtained in the presence of inhibitor fall significantly below that of free acid. In all cases, the increase in the inhibitor concentration was accompanied by a decrease in weight loss and an increase in the percentage inhibition. These results lead to the conclusion that the compound under investigation is fairly efficient as inhibitors for low-carbon steel dissolution in hydrochloric acid solution. Also, the degree of surface coverage $(\theta)$ by the inhibitor would increase by increasing the inhibitor concentration. In order to get a comparative view, the variation of the percentage inhibition (\% IE) of the inhibitor with its concentrations was calculated and its values obtained are summarized in Table 1 .

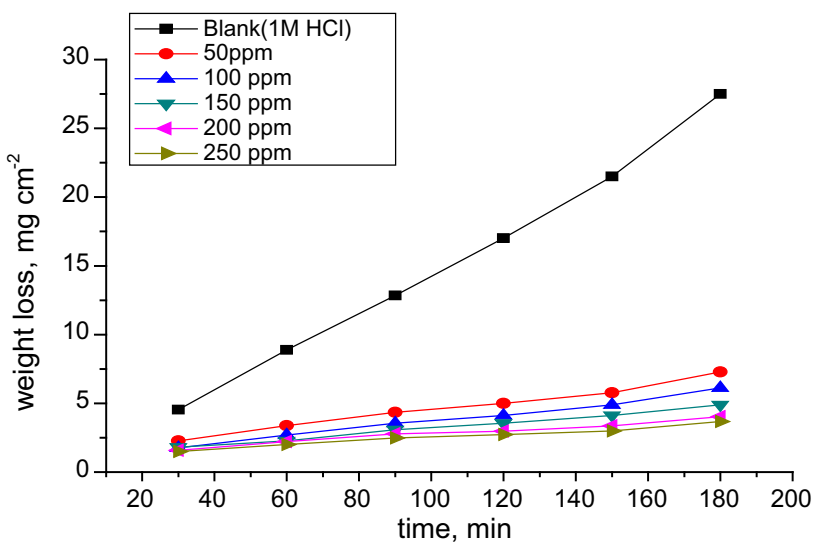

Fig. 1 Weight loss-time curves for the corrosion of low-carbon steel in $1 \mathrm{M} \mathrm{HCl}$ in the absence and presence of different concentrations of Amlodipine Besylate at $30{ }^{\circ} \mathrm{C}$

Table 1 Inhibition efficiency and corrosion rate (C.R) of Amlodipine Besylate for the corrosion of low-carbon steel in $1 \mathrm{M} \mathrm{HCl}$ from weight loss measurements at different concentrations and at $30^{\circ} \mathrm{C}$

\begin{tabular}{|c|c|c|c|}
\hline [Inh] ppm & C.R $\mathrm{mg} \mathrm{cm} \mathrm{cm}^{-2} \min ^{-1}$ & $\theta$ & $\% \mathrm{IE}$ \\
\hline Blank & 0.1420 & - & - \\
\hline 50 & 0.0420 & 0.706 & 70.6 \\
\hline 100 & 0.0283 & 0.758 & 75.8 \\
\hline 150 & 0.0235 & 0.791 & 79.1 \\
\hline 200 & 0.0220 & 0.825 & 82.5 \\
\hline 250 & 0.0227 & 0.840 & 84.0 \\
\hline
\end{tabular}

\subsection{Effect of Temperature}

The effect of temperature on the corrosion rate of low-carbon steel in $1 \mathrm{M} \mathrm{HCl}$ and in the presence of different inhibitor concentrations was studied at two temperatures $\left(30\right.$ and $45{ }^{\circ} \mathrm{C}$ ) using weight loss measurements. As the temperature increases, the rate of corrosion increases and the inhibition efficiency of Amlodipine Besylate decreases as shown in Table 2. The adsorption behavior of Amlodipine Besylate on $\mathrm{HCl}$ surface occurs through physical adsorption.

\subsection{Adsorption Isotherms}

One of the most convenient ways of expressing adsorption quantitatively is by deriving the adsorption isotherm that characterizes the metal/inhibitor/environment system. Various adsorption isotherms were applied to fit $\Theta$ values, but the best fit was found to obey Langmuir adsorption isotherm which is represented in Fig. 2 for Amlodipine Besylate, and Langmuir adsorption isotherm may be expressed as 
Table 2 Data of weight loss measurements for low-carbon steel in $1 \mathrm{M} \mathrm{HCl}$ solution in the absence and presence of different concentrations of Amlodipine Besylate at 30 and $45^{\circ} \mathrm{C}$

\begin{tabular}{|c|c|c|c|c|}
\hline \multirow[t]{2}{*}{ [Inh] ppm } & \multicolumn{2}{|l|}{$30^{\circ} \mathrm{C}$} & \multicolumn{2}{|l|}{$45^{\circ} \mathrm{C}$} \\
\hline & C.R $\mathrm{mg} \mathrm{cm}{ }^{-2} \mathrm{~min}^{-1}$ & $\begin{array}{l}\% \\
\text { IE }\end{array}$ & C.R $\mathrm{mg} \mathrm{cm}{ }^{-2} \mathrm{~min}^{-1}$ & $\begin{array}{l}\% \\
\text { IE }\end{array}$ \\
\hline Blank & 0.1420 & - & 0.46 & - \\
\hline 50 & 0.0420 & 70.6 & 0.23 & 49.9 \\
\hline 100 & 0.0283 & 75.8 & 0.21 & 54.2 \\
\hline 150 & 0.0235 & 79.1 & 0.17 & 62.5 \\
\hline 200 & 0.0220 & 82.5 & 0.16 & 65.8 \\
\hline 250 & 0.0227 & 84.0 & 0.14 & 69.0 \\
\hline
\end{tabular}

$(C / \theta)=1 / \operatorname{Kads}+C$,

where $C$ is the concentration $\left(\mathrm{mol} \mathrm{L}^{-1}\right)$ of the inhibitor in the bulk electrolyte, $\theta$ is the degree of surface coverage $(\theta=\% \mathrm{IE} / 100)$, and $K_{\mathrm{ads}}$ is the adsorption equilibrium constant. A plot of $\mathrm{C}$ versus $C / \theta$ should give straight line. In order to get a comparative view, the variation of the adsorption equilibrium constant $\left(K_{\mathrm{ads}}\right)$ of the Amlodipine Besylate with its concentration was calculated. The experimental data give good curve fitting for the applied adsorption isotherm as the correlation coefficients $\left(R^{2}\right)$ were more than 0.99 . The values obtained are shown in Table 3. These results confirm the assumption that this compound is adsorbed on the metal surface through the protonated $(\mathrm{N}, \mathrm{O})$ atoms or via the lone pair of electrons of $(\mathrm{N}, \mathrm{O})$ atoms. The extent of inhibition is directly related to the performance of adsorption layer which is a sensitive function of the molecular structure. The equilibrium constant of adsorption $K_{\text {ads }}$ obtained from the intercept of Langmuir adsorption isotherm is related to the free energy of adsorption $\Delta G^{\circ}$ ads as follows:

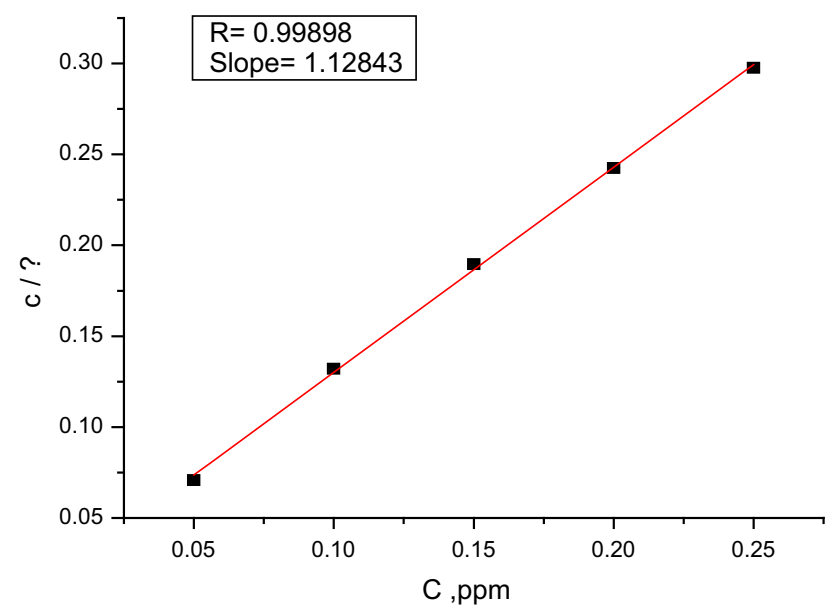

Fig. 2 Langmuir adsorption isotherm for Amlodipine Besylate drug
Table 3 Thermodynamic parameters for the adsorption on low-carbon steel surface in $1 \mathrm{M} \mathrm{HCl}$ at different temperatures

\begin{tabular}{|c|c|c|c|c|}
\hline Temp. ${ }^{\circ} \mathrm{C}$ & $K_{\text {ads }} \mathrm{M}^{-1}$ & $-\Delta G_{\text {ads }}^{\circ} \mathrm{kJ} \mathrm{mol}^{-1}$ & $Q_{\text {ads }} \mathrm{kJ} \mathrm{mol}^{-1}$ & $R^{2}$ \\
\hline 30 & 57.971 & 20.3 & - & 0.999 \\
\hline 45 & 21.857 & 18.8 & 45.4 & 0.992 \\
\hline
\end{tabular}

$\left.K_{\text {ads }}=1 / 55.5 \exp \left[-\Delta G_{\text {ads }}^{\circ}\right] / R T\right]$,

where 55.5 is the molar concentration of water in the solution in $\mathrm{M}^{-1}$.

Table 3 clearly shows a good dependence of $\Delta G^{\circ}$ ads on $\mathrm{T}$, indicating the good correlation among thermodynamic parameters. The negative value of $\Delta G^{\circ}$ ads reflects that the adsorption of the studied compound on low-carbon steel surface from $1 \mathrm{M} \mathrm{HCl}$ solution is spontaneous process and stability of the adsorbed layer on the low-carbon steel surface. Generally, the values of $\Delta G^{\circ}$ ads around $-20 \mathrm{~kJ} \mathrm{~mol}^{-1}$ or lower are consistent with the electrostatic interaction between the charged molecules and the charged metal (physical adsorption); those around $-40 \mathrm{~kJ} \mathrm{~mol}^{-1}$ or higher involve charge sharing or transfer from organic molecules to the metal surface to form a coordinate type of bond (chemisorption) [27]. From the obtained values of $\Delta G$ ${ }^{\circ}{ }_{\text {ads }}$, the existence of physical adsorption was observed. The unshared electron pairs in oxygen and nitrogen may interact with d-orbitals of low-carbon steel to provide a protective physical adsorbed film [28]. The values of thermodynamic parameters for the adsorption of tested compound can provide valuable information about the mechanism of corrosion inhibition. An estimate of heat of adsorption was obtained from the trend of surface coverage with temperature as follows [29]:

$$
\begin{aligned}
Q_{\mathrm{ads}}= & 2.303 R\left[\log \left(\frac{\theta_{2}}{1-\theta_{2}}\right)-\log \left(\frac{\theta_{1}}{1-\theta_{1}}\right)\right] \\
& \times\left(\frac{T_{1} \times T_{2}}{T_{2}-T_{1}}\right),
\end{aligned}
$$

where $\theta_{1}$ and $\theta_{2}$ are the degree of surface coverage at temperature $T_{1}$ and $T_{2}$. respectively. The calculated values for both parameters are shown in Table 3 .

\subsection{Kinetic-Thermodynamic Corrosion Parameters}

The effect of temperature on corrosion inhibition of lowcarbon steel in $1 \mathrm{M} \mathrm{HCl}$ solution in the absence and presence of different concentrations of the investigated compound at different temperatures of 30 and $45{ }^{\circ} \mathrm{C}$ was studied using weight loss measurements. The corrosion rate was found to increase with increasing temperature both in uninhibited and inhibited solutions. The apparent activation energy $\left(E_{\mathrm{a}}^{*}\right)$ for the corrosion process can be calculated from Arrhenius-type equation: 
$\log \rho_{2} / \rho_{1}=\left(E_{a}^{*} / 2.303 R\right) \times\left(\frac{1}{T_{1}}-\frac{1}{T_{2}}\right)$,

where $E_{\mathrm{a}}^{*}$ is the apparent activation corrosion energy, $R$ is the universal gas constant, and $\rho_{1}$ and $\rho_{2}$ are the corrosion rates at temperature $T_{1}$ and $T_{2}$, respectively

The calculated values of $E_{\mathrm{a}}^{*}$ are $63.5 \mathrm{~kJ} \mathrm{~mol}^{-1}$ for blank and $72.2 \mathrm{~kJ} \mathrm{~mol}^{-1}$ in the presence of the investigated compound. The value of $E_{\mathrm{a}}^{*}$ determined in solution containing the investigated compound is higher than that in its absence.

The decrease in \%IE values as the temperature is increased and high values of $E_{\mathrm{a}}^{*}$ in the presence of the investigated compound can be interpreted as an indication for a physical or columbic type of adsorption [30]. The high $E_{\mathrm{a}}^{*}$ value in the inhibited solution can be correlated with the increased thickness of the double layer, which enhances the activation energy of the corrosion process.

\subsection{Potentiodynamic Polarization Tests}

Figures 3 shows typical polarization curves for low-carbon steel in $1 \mathrm{M} \mathrm{HCl}$ media. In the presence of Amlodipine Besylate, both the cathodic and anodic current densities were greatly decreased over a wide potential range. Various corrosion parameters such as corrosion potential $\left(E_{\text {corr }}\right)$, anodic and cathodic Tafel slopes $\left(\beta_{\mathrm{a}}, \beta_{\mathrm{c}}\right)$, corrosion current density $\left(i_{\text {corr }}\right)$, degree of surface coverage $(\theta)$, and inhibition efficiency (\%IE) are shown in Table 4. It can be seen from the experimental results that in all cases the addition of Amlodipine

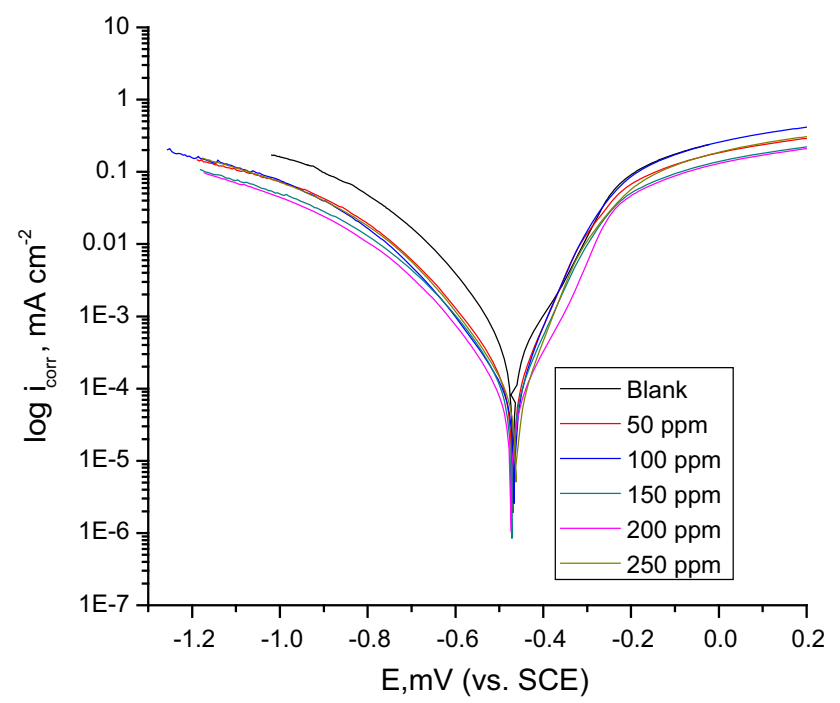

Fig. 3 Potentiodynamic polarization curves for the dissolution of low-carbon steel in $1 \mathrm{M} \mathrm{HCl}$ in the absence and presence of different concentrations of Amlodipine Besylate at $30{ }^{\circ} \mathrm{C}$
Besylate induced a significant decrease in cathodic and anodic currents. The values of $E_{\text {corr }}$ were affected and slightly changed by the addition of Amlodipine Besylate. This indicates that Amlodipine Besylate acts as a mixedtype inhibitor. The slopes of anodic and cathodic Tafel lines $\left(\beta_{\mathrm{a}}\right.$ and $\left.\beta_{\mathrm{c}}\right)$ were slightly changed (Tafel lines are parallel) on increasing the concentration of the tested compound, which indicates that there is no change in the mechanism of inhibition in the presence and absence of Amlodipine Besylate. The order of inhibition efficiency of Amlodipine Besylate at different concentrations as observed by polarization measurements are listed in Table 4. The results are in good agreement with those obtained from weight loss measurements.

\subsection{Electrochemical Impedance Spectroscopy (EIS) Tests}

EIS is well established and is a powerful technique for studying the corrosion. Surface properties, electrode kinetics, and mechanistic information can be obtained from impedance diagrams. Figure 4 shows the Nyquist (a) and Bode (b) plots obtained at open circuit potential. The increase in the size of the capacitive loop with the addition of investigated compound shows that a barrier gradually forms on the low-carbon steel surface (Fig. 4a). Bode plot (Fig. 4b) shows that the total impedance increases with increasing inhibitor concentration ( $\log Z$ vs. $\log f$ ) except for ( $\log f$ vs. phase). Bode plot also shows the continuous increase in the phase angle shift, obviously correlating with the increase of inhibitor adsorbed on low-carbon steel surface. The Nyquist plot does not yield perfect semicircles as expected from the theory of EIS. The deviation from ideal semicircle was generally attributed to the frequency dispersion [31] as well as to the inhomogeneities of the surface. EIS spectra of the investigated compounds were analyzed using the equivalent circuit (Fig. 5), which represents a single charge transfer reaction and fits well with our experimental results. The constant phase element, CPE, is introduced in the circuit instead of a pure double-layer capacitor to give a more accurate fit [32].The double-layer capacitance, $C_{\mathrm{dl}}$, for a circuit including a CPE parameter $\left(Y_{0}\right.$ and $n$ ) was calculated using the following equation [33]:

$C_{\mathrm{dl}}=Y_{0}\left(\omega_{\max }\right)^{n-1}$,

where $Y_{0}$ is the magnitude of the CPE, $\omega_{\max }=2 \pi f_{\max }, f_{\max }$ is the frequency at which the imaginary component of the impedance is maximal, and the factor $\mathrm{n}$ is an adjustable parameter that usually lies between 0.50 and 1.0. After analyzing the shape of the Nyquist plots, it is concluded that the curves approximated by single capacitive 
Table 4 Corrosion potential $\left(E_{\text {corr }}\right)$, corrosion current density $\left(i_{\text {corr }}\right)$, Tafel slopes $\left(\beta_{\mathrm{c}}, \beta_{\mathrm{a}}\right)$,corrosion rate (CR), degree of surface coverage $(\theta)$, and inhibition efficiency (\% IE) of low-carbon steel in $1 \mathrm{M} \mathrm{HCl}$ at

\begin{tabular}{|c|c|c|c|c|c|c|c|}
\hline [Inh.] ppm & $-\mathrm{E}_{\text {corr }} \mathrm{mV}$ versus $\mathrm{SCE}$ & $i_{\text {corr }} \times 10^{-4} \mu \mathrm{A} \mathrm{cm}^{-2}$ & $\beta_{\mathrm{c}} \mathrm{mV} \mathrm{dec}{ }^{-1}$ & $\beta_{\mathrm{a}} \mathrm{mV} \mathrm{dec}{ }^{-1}$ & CR mpy & $\theta$ & $\% \mathrm{IE}$ \\
\hline Blank & 469 & 301.0 & 117 & 116 & 137.40 & - & - \\
\hline 50 & 470 & 105.0 & 118 & 79 & 47.82 & 0.651 & 65.1 \\
\hline 100 & 466 & 85.9 & 128 & 71 & 39.24 & 0.715 & 71.5 \\
\hline 150 & 471 & 84.5 & 120 & 83 & 38.59 & 0.719 & 71.9 \\
\hline 200 & 472 & 63.1 & 118 & 96 & 28.83 & 0.790 & 79.0 \\
\hline 250 & 462 & 49.7 & 79 & 63 & 22.70 & 0.835 & 83.5 \\
\hline
\end{tabular}
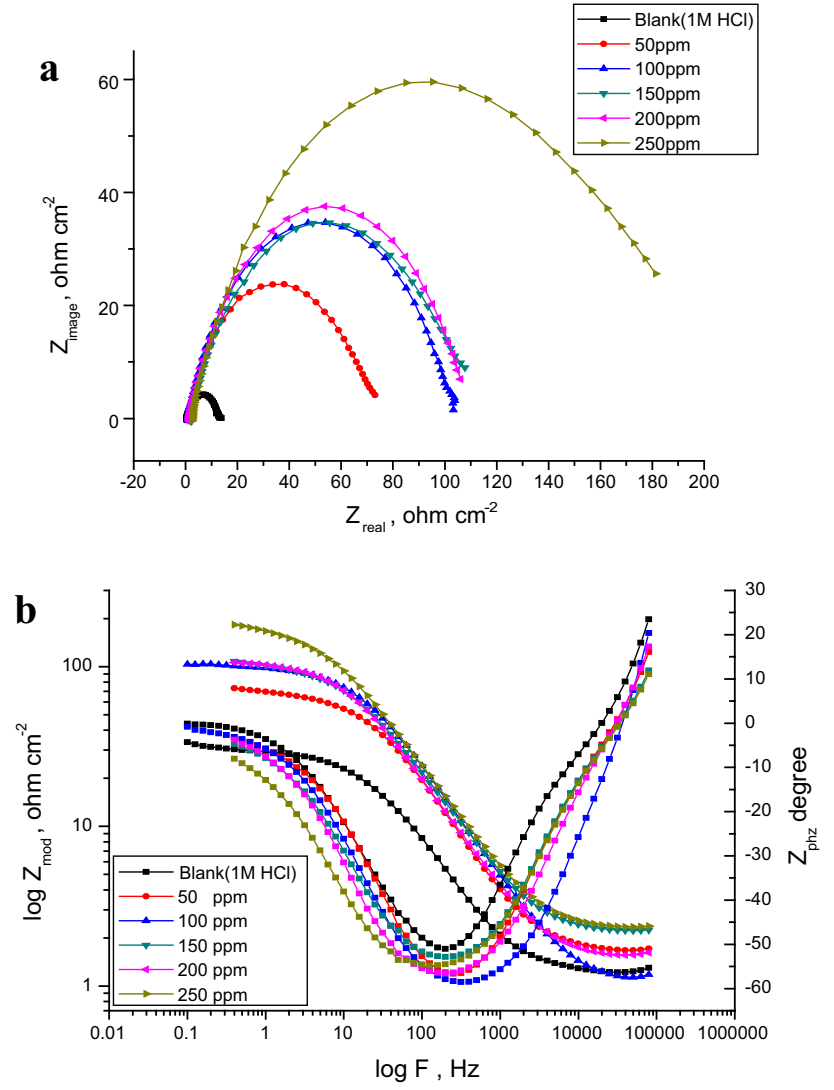

Fig. 4 a Nyquist plots for the corrosion of low-carbon steel in $1 \mathrm{M}$ $\mathrm{HCl}$ in the absence and presence of different concentrations of Amlodipine Besylate at $30^{\circ} \mathrm{C}$. b Bode plots for the corrosion of lowcarbon steel in $1 \mathrm{M} \mathrm{HCl}$ in the absence and presence of different concentrations of Amlodipine Besylate at $30{ }^{\circ} \mathrm{C}$

semicircles show that the corrosion process was mainly charge transfer controlled [34, 35]. The general shape of the curves is very similar for all samples (in the presence or absence of inhibitor for different immersion times) indicating no change in the corrosion mechanism [36]. From the impedance data in Table 5, we conclude that the value of $R_{\mathrm{ct}}$ increases with increasing the concentration of
$30{ }^{\circ} \mathrm{C}$ for Amlodipine Besylate in the absence and presence of increasing concentrations of investigated compound at $30{ }^{\circ} \mathrm{C}$ 
$\% I E_{\mathrm{EIS}}=\left[1-\left(R_{\mathrm{ct}}^{\circ} / R_{\mathrm{ct}}\right)\right] \times 100$,

where $R_{\text {ct }}^{\circ}$ and $R_{\text {ct }}$ are the charge transfer resistance values without and with inhibitor, respectively.

\subsection{Electrochemical Frequency Modulation (EFM) Tests}

EFM is a nondestructive corrosion measurement technique that can directly and quickly determine the corrosion current values without prior knowledge of Tafel slopes, and with only a small polarizing signal. These advantages of EFM technique make it an ideal candidate for online corrosion monitoring [39]. The great strength of the EFM is the causality factors which serve as an internal check on the validity of EFM measurement. The causality factors CF-2 and CF-3 are calculated from the frequency spectrum of the current responses. Figure 6 shows the EFM intermodulation spectra (current vs frequency) of low-carbon steel in $\mathrm{HCl}$ solution containing different concentrations of Amlodipine Besylate. The harmonic and intermodulation peaks are clearly visible and are much larger than the background noise. The larger peaks were used to calculate the corrosion current density $\left(i_{\text {corr }}\right)$, the Tafel slopes $\left(\beta_{\mathrm{c}}\right.$ and $\beta_{\mathrm{a}}$ ), and the causality factors (CF-2 and CF-3). These electrochemical parameters are listed in Table 6 . The data presented in Table 6 obviously show that the addition of the tested compound at a given concentration to the acidic solution decreases the corrosion current density, indicating that this compound inhibits the corrosion of low-carbon steel in $1 \mathrm{M} \mathrm{HCl}$ through adsorption. The causality factors obtained under different experimental conditions are approximately equal to the theoretical values (2 and 3 ) indicating that the measured data are verified and of good quality. The inhibition efficiencies $\% \mathrm{IE}_{\mathrm{EFM}}$ increase by increasing the inhibitor concentration and were calculated using Eq. 2.

\subsection{Surface Examinations}

Scanning electron microscope (SEM) and energy-dispersive X-ray (EDX) experiments were carried out in order to verify if the investigated compound is in fact adsorbed on low-carbon steel surface or just peeled off the surface. SEM images were indicative of the changes that accompany both corrosion and protection of the low-carbon steel surface (Fig. 7a-c). Figure 7a shows the free metal, while Fig. $7 \mathrm{~b}$ shows the damage caused to the surface by hydrochloric acid. Figure 7c shows SEM images of the low-carbon steel surface after treatment with $1 \mathrm{M} \mathrm{HCl}$ containing $150 \mathrm{ppm}$ of inhibitor. From these images, it is obvious that the steel surface seems to be almost unaffected by corrosion. This is because of adsorption of inhibitor, forming a thin protective film of the inhibitor on the metal surface. This film is responsible for the highly efficient inhibition by this inhibitor. The corresponding EDX profile
Fig. 6 EFM spectra for lowcarbon steel in $1 \mathrm{M} \mathrm{HCl}$ in the absence and presence of different concentrations of Amlodipine Besylate at $30^{\circ} \mathrm{C}$
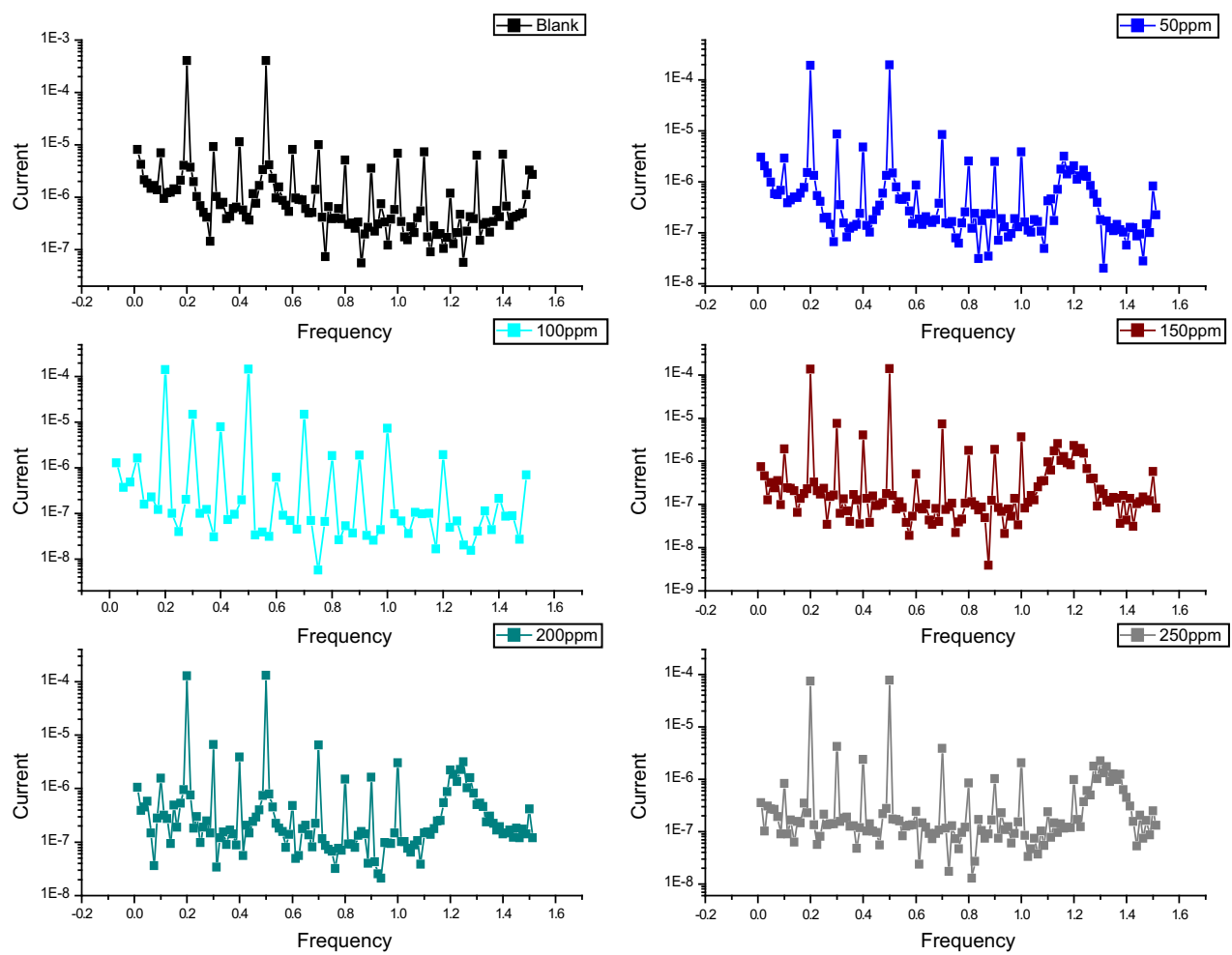
Table 6 Electrochemical kinetic parameters obtained by EFM technique for low-carbon steel in $1 \mathrm{M} \mathrm{HCl}$ in the absence and presence of different concentrations of Amlodipine Besylate at $30^{\circ} \mathrm{C}$

\begin{tabular}{lllllllll}
\hline [Inh] $\mathrm{ppm}$ & $i_{\text {corr }}\left(\mu \mathrm{A} \mathrm{cm}^{2}\right)$ & $\beta_{\mathrm{a}}\left(\mathrm{mV} \mathrm{dec}^{-1}\right)$ & $\beta_{\mathrm{c}}\left(\mathrm{mV} \mathrm{dec}^{-1}\right)$ & $\mathrm{CF}-2$ & $\mathrm{CF}-3$ & $\mathrm{CR} \mathrm{mpy}$ & $\theta$ & $\% \mathrm{IE}$ \\
\hline Blank & 700.4 & 104.8 & 124.0 & 1.94 & 2.79 & 7.37 & & - \\
50 & 308.8 & 91.45 & 121.0 & 1.92 & 2.88 & 0.23 & 0.60 & 60 \\
100 & 274.4 & 89.14 & 204.4 & 1.97 & 3.17 & 1.76 & 0.61 & 61 \\
150 & 210.2 & 85.76 & 120.3 & 1.94 & 2.77 & 0.62 & 0.70 & 70 \\
200 & 205.7 & 89.04 & 123.3 & 1.95 & 3.12 & 1.26 & 0.71 & 71 \\
250 & 127.9 & 93.02 & 133.5 & 1.94 & 2.85 & 0.81 & 0.82 & 82 \\
\hline
\end{tabular}

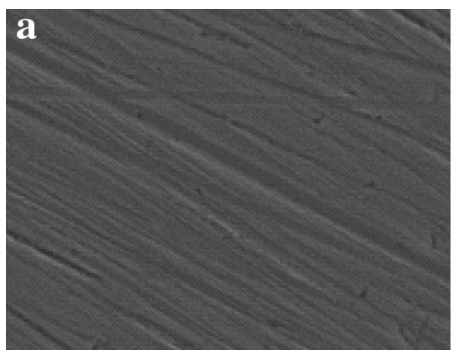

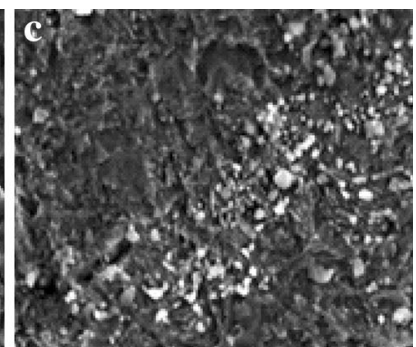

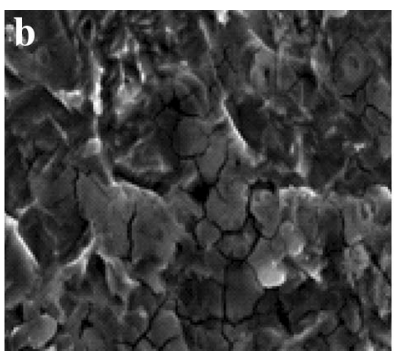

Fig. 7 SEM micrographs of low-carbon steel surface a before immersion in $1 \mathrm{M} \mathrm{HCl}, \mathbf{b}$ after $24 \mathrm{~h}$ of immersion in $1 \mathrm{M}$ $\mathrm{HCl}$, and $\mathbf{c}$ after $24 \mathrm{~h}$ of immersion in $1 \mathrm{M}$ $\mathrm{HCl}+150 \mathrm{ppm}$ of Amlodipine Besylate analyses are presented in Fig. 7 for the investigated drug. It is also important to notice the existence of $\mathrm{C}, \mathrm{N}$, and $\mathrm{O}$ peaks in the EDX spectra of the low-carbon steel surface corresponding to the samples immersed for 1 day in solutions containing $150 \mathrm{ppm}$ of this drug. The formation of a thin inhibitor film is in agreement with the SEM observations (Fig. 8).

The results in Table 7 showed that the $\%$ weight of $\mathrm{Fe}$ in the presence of the drug is decreased and $\%$ weight of $\mathrm{C}$, $\mathrm{O}$, and $\mathrm{N}$ atoms increased. This is due to the film formation of the drug on the low-carbon steel surface.

\subsection{Quantum Calculations}

The energy of highest occupied molecular orbital ( $\left.E_{\mathrm{HOMO}}\right)$ indicates the ability of the molecule to donate electrons $(-8.962 \mathrm{eV})$ to an appropriate acceptor with empty molecular orbitals, whereas the energy of unoccupied molecular orbital $\left(E_{\mathrm{LUMO}}\right)$ indicates its ability to accept electrons $(-0.472 \mathrm{eV})$. The lower the value of $E_{\mathrm{LUMO}}$, the more the ability of the molecule to accept electrons [40]. The higher the value of $E_{\mathrm{HOMO}}$ of the inhibitor, the easier is its ability to offer electrons to the unoccupied d-orbital of metal surface and the greater is its inhibition efficiency.

The $E_{\text {HOMO }}-E_{\text {LUMO }}$ energy gap, $\Delta E$ approach, $(8.490 \mathrm{eV})$ which is an important stability index, is applied to develop theoretical models for explaining the structure and conformation barriers in many molecular systems. The smaller the value of $\Delta E$, the more is the probable inhibition efficiency the compound [41]. Variation in the inhibition efficiency of the inhibitor depends on the presence of electronegative $\mathrm{O}$ and $\mathrm{N}$ atoms as a substituent in their molecular structure. The calculated charges of selected atoms are presented in Fig. 9.

\subsection{Mechanism of Corrosion Inhibition}

The inhibition mechanism involves the adsorption of the inhibitor on the metal surface immersed in aqueous $\mathrm{HCl}$ solution. Four types of adsorption [42] may take place involving organic molecules at the metal-solution interface: (1) electrostatic attraction between the charged molecules and the charged metal; (2) interaction of unshared electron pairs in the molecule with the metal; (3) interaction of $\pi$-electrons with the metal; and (4) combination of all the above. From the observations drawn from the different methods, corrosion inhibition of low-carbon steel in $1 \mathrm{M} \mathrm{HCl}$ solutions by the investigated inhibitor as indicated from weight loss, potentiodynamic polarization, and EIS techniques was found to depend on the concentration and the nature of the inhibitor. Amlodipine Besylate contains polar groups such as oxygen and nitrogen. Each atom is a chemisorption center and the inhibition efficiency depends on the electron density around the chemisorption center; the higher the electron density at the chemisorption center, the greater is the inhibition efficiency. This inhibitor has high inhibition efficiency as it has oxygen and $\mathrm{N}$ atoms with lone pair of electrons. These electrons interact with the vacant d-orbital of iron present in the low-carbon steel surface and adsorb strongly thereby blocking more number 

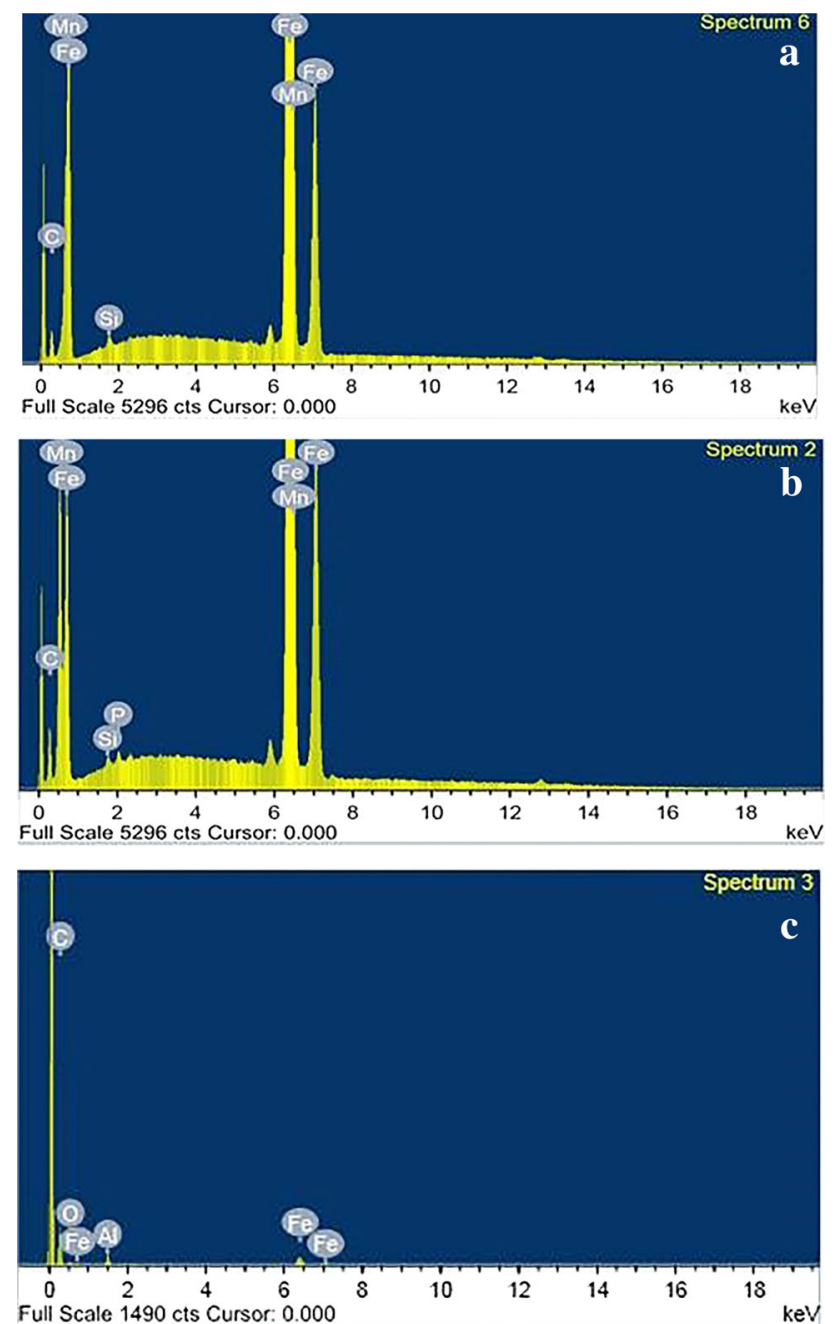

Fig. 8 EDX spectra of low-carbon steel in $1 \mathrm{M} \mathrm{HCl}$ a before immersion in $1 \mathrm{M} \mathrm{HCl}$, b after $24 \mathrm{~h}$ of immersion in $1 \mathrm{M} \mathrm{HCl}$, and c after $24 \mathrm{~h}$ of immersion in $1 \mathrm{M} \mathrm{HCl}+150 \mathrm{ppm}$ of Amlodipine Besylate

Table 7 Surface composition (wt \%) of low-carbon steel after $24 \mathrm{~h}$ of immersion in $1 \mathrm{M} \mathrm{HCl}$ without and with $150 \mathrm{ppm}$ of the studied drug

\begin{tabular}{lllll}
\hline Mass $\%$ & $\mathrm{Fe}$ & $\mathrm{C}$ & $\mathrm{O}$ & $\mathrm{N}$ \\
\hline Pure & 87.72 & 12.28 & - & - \\
Blank & 82.06 & 17.94 & - & - \\
$150 \mathrm{ppm}$ & 65.02 & 23.28 & 17.8 & 11.70 \\
\hline
\end{tabular}

of adsorption sites on the low-carbon steel surface. The presence of oxygen and nitrogen caused a resonance effect which facilitates stronger adsorption of inhibitor on lowcarbon steel surface. This leads to high IE for Amlodipine Besylate. It has been previously reported in literature that the inhibiting effect depends mainly on inhibitor concentration, molecular structure, size, and structure of the side chain in the organic compounds.

\section{HOMO}
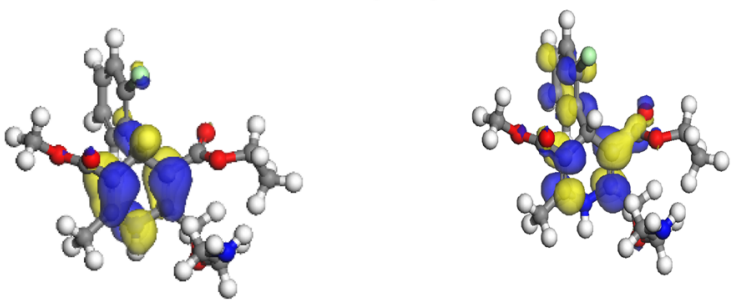

LUMO
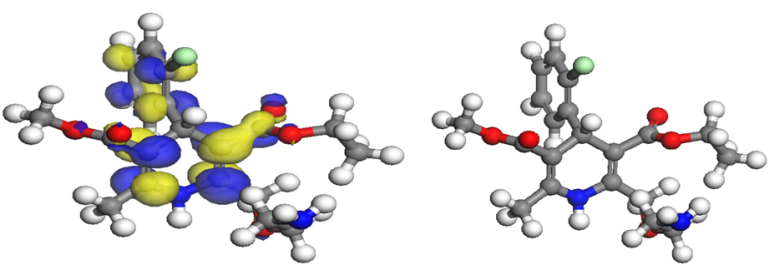

Fig. 9 Optimized molecular structure of inhibitor and their frontier molecular orbital density distribution (HOMO and LUMO)

\section{Conclusions}

From the overall experimental results, the following conclusions can be deduced:

1. The investigated compound is a good inhibitor and acts as a mixed-type inhibitor for low-carbon steel corrosion in $1 \mathrm{M} \mathrm{HCl}$ solution.

2. Reasonably good agreement was observed between the values obtained by the weight loss and electrochemical measurements.

3. The results obtained from all measurements showed that the inhibition efficiency increases with the increase in the inhibitor concentration and decreases with the rise in temperature.

4. Double-layer capacitances decrease with respect to blank solution when the inhibitor is added. This fact confirms the adsorption of this molecule on the lowcarbon steel surface.

5. The thermodynamic parameters revealed that the inhibition of corrosion by the investigated compound is due to the formation of a physical adsorbed film on the metal surface.

6. The adsorption of inhibitor on low-carbon steel surface in $\mathrm{HCl}$ solution follows Langmuir isotherm for the compound.

7. The values of inhibition efficiency obtained from the different independent quantitative techniques used show the validity of the results.

8. Quantum chemical parameters for this investigated compound were calculated to provide further insight into the mechanism of inhibition of the corrosion process. 


\section{References}

1. Tianbao Du, Chen Jiajian, Cao Dianzhen (2001) N,N-Dipropynoxy methyl amine trimethyl phosphonate as corrosion inhibitor for iron in sulfuric acid. J Mater Sci 36:3903-3907

2. Morales-Gil P, Negron-Silva G, Romero-Romo M, AngelesChavez C, Palomar-Pardave M (2004) Corrosion inhibition of pipeline steel grade API 5L X52 immersed in a $1 \mathrm{M} \mathrm{H}_{2} \mathrm{SO}_{4}$ aqueous solution using heterocyclic organic molecules. Electrochim Acta 49:4733-4741

3. Bastidas JM, Polo JL, Cano E (2000) Substitutional inhibition mechanism of mild steel hydrochloric acid corrosion by hexylamine and dodecylamine. J Appl Electrochem 30:1173-1177

4. Zerga B, Attiyibat A, Sfaira M, Taleb M, Hammouti B, Ebn Touhami M, Radi S, Rais Z (2010) Effect of some tripodal bipyrazolic compounds on $\mathrm{C} 38$ steel corrosion in hydrochloric acid solution. J Appl Electrochem 40:1575-1582

5. Tamil Selvi S, Raman V, Rajendran N (2003) Corrosion inhibition of mild steel by benzotriazole derivatives in acidic medium. $\mathrm{J}$ Appl Electrochem 33:1175-1182

6. Lowmunkhong P, Ungthararak D, Sutthivaiyakit P (2010) Tryptamine as a corrosion inhibitor of mild steel in hydrochloric acid solution. Corros Sci 52:30-36

7. Zerfaoui M, Oudda H, Hammouti B, Kertit S, Benkaddour M (2004) Inhibition of corrosion of iron in citric acid media by aminoacids. Prog Org Coat 51:134-138

8. Chetaouani A, Hammouti B, Aouniti A, Benchat N, Benhadda T (2002) New synthesised pyridazine derivatives as effective inhibitors for the corrosion of pure iron in $\mathrm{HCl}$ medium. Prog Org Coat 45:373-378

9. Mohamed Awad K (2004) Semiempirical investigation of the inhibition efficiency of thiourea derivatives as corrosion inhibitors. J Electroanal Chem 567:219-225

10. Yadav DK, Maiti B, Quraishi MA (2010) Electrochemical and quantum chemical studies of 3,4-dihydropyrimidin-2(1H)-ones as corrosion inhibitors for mild steel in hydrochloric acid solution. Corros Sci 52:3586-3598

11. Jacob KS, Parameswaran G (2010) Corrosion inhibition of mild steel in hydrochloric acid solution by Schiff base furoin thiosemicarbazone. Corros Sci 52:224-228

12. Ostovari A, Hoseinieh SM, Peikari M, Shadizadeh SR, Hashemi SJ (2009) Corrosion inhibition of mild steel in $1 \mathrm{M} \mathrm{HCl}$ solution by henna extract: A comparative study of the inhibition by henna and its constituents (Lawsone, Gallic acid, $\alpha$-D-Glucose and Tannic acid). Corros Sci 51:1935-1949

13. Giacomelli FC, Giacomelli C, Amadori MF, Schmidt V, Spinelli A (2004) Inhibitor effect of succinic acid on the corrosion resistance of mild steel: electrochemical, gravimetric and optical microscopic studies. Mater Chem Phys 83:124-128

14. Satapathy AK, Gunasekaran G, Sahoo SC, Amit K, Rodrigues PV (2009) Corrosion inhibition by Justicia gendarussa plant extract in hydrochloric acid solution. Corros Sci 51:2848-2856

15. Gebhart F (2005) Drug Topics, October 10 (http://drugtopics. modernmedicine.com/drugtopics/article/articleDetail.jsp?id=184115. Accessed 17 Feb 2012)

16. Vaszilcsin N, Ordodi V, Borza A (2012) Corrosion inhibitors from expired drugs. Int J Pharm 431:241-244

17. Obot IB, Obi-Egbedi NO, Umoren SA (2009) Antifungal drugs as corrosion inhibitors for aluminium in $0.1 \mathrm{M} \mathrm{HCl}$. Corros Sci $51: 1868-1875$

18. Abdallah M (2004) Antibacterial drugs as corrosion inhibitors for corrosion of aluminium in hydrochloric solution. Corros Sci 46:1981-1996
19. Fouda AS, Al-Sarawy AA, Ahmed FSh, El-Abbasy HM (2009) Corrosion inhibition of aluminum 6063 using some pharmaceutical compounds. Corros Sci 51:485-492

20. Obot IB, Obi-Egbedi NO (2008) inhibitory effect and adsorption characteristics of 2, 3-diaminonaphthalene at aluminum/hydrochloric acid interface: experimental and theoretical study. Surf Rev Lett 15:903-910

21. Fouda AS, EL-Haddad MN, Abdallah YM (2013) Septazole: antibacterial drug as a green corrosion inhibitor for copper in hydrochloric acid solutions. Int J Innov Res Sci Eng Technol 2 (12):7073-7085

22. Imran Naqvi AR, Saleemi Naveed S (2011) Cefixime: a drug as efficient corrosion inhibitor for low carbon steel in acidic media. Electrochemical and thermodynamic studies. Int J Electrochem Sci 6:146-161

23. Oguzie EE (2005) Corrosion inhibition of mild steel in hydrochloric acid solution by methylene blue dye. Mater Lett 59:1076-1079

24. Khaled KF (2008) Application of electrochemical frequency modulation for monitoring corrosion and corrosion inhibition of iron by some indole derivatives in molar hydrochloric acid. Mater Chem Phys 112:290-300

25. Khaled KF (2009) Evaluation of electrochemical frequency modulation as a new technique for monitoring corrosion and corrosion inhibition of carbon steel in perchloric acid using hydrazine carbodithioic acid derivatives. J Appl Electrochem 39:429-438

26. Abdel-Rehim SS, Khaled KF, Abd-Elshafi NS (2006) Electrochemical frequency modulation as a new technique for monitoring corrosion inhibition of iron in acid media by new thiourea derivative. Electrochim Acta 5:3269-3277

27. Yurt A, Bereket G, Kivrak A, Balaban A, Erk B (2005) Effect of schiff bases containing pyridyl group as corrosion inhibitors for low carbon steel in 0.1 M HCl. J Appl Electrochem 35:10251032

28. Bentiss F, Traisnel M, Lagrenee M (2000) The substituted 1,3,4oxadiazoles: a new class of corrosion inhibitors of mild steel in acidic media. Corros Sci 42:127-146

29. Gao G, Liang C (2007) Electrochemical and DFT studies of $\beta$ amino-alcohols as corrosion inhibitors for brass. Electrochim Acta 52:4554-4559

30. Quraishi MA, Jamal D (2003) Dianils as new and effective corrosion inhibitors for mild steel in acidic solutions Mater. Chem Phys 78:608-613

31. Schmid GM, Huang HJ (1980) Spectro-electrochemical studies of the inhibition effect of 4,7-diphenyl -1, 10-phenanthroline on the corrosion of 304 stainless steel. Corros Sci 20:1041-1057

32. Bentiss F, Lebrini M, Lagrenee M (2005) Thermodynamic characterization of metal dissolution and inhibitor adsorption processes in mild steel/2,5-bis(n-thienyl)-1,3,4-thiadiazoles/hydrochloric acid system. Corros Sci 47:2915-2931

33. Marsh J (1988) Advanced organic chemistry, 3rd edn. Wieley Eastern, New Delhi

34. Silverman DC, Carrico JE (1988) Electrochemical impedance technique-a practical tool for corrosion prediction. Corrosion 44:280-287

35. Lorenz WJ, Mansfeld F (1981) Determination of corrosion rates by electrochemical DC and AC methods. Corros Sci 21:647-672

36. Gadiyar HS, ELayathu NSD (1980) Corrosion and magnetite growth on carbon steels in water at $310 \mathrm{C}$-effect of dissolved oxygen, $\mathrm{pH}$, and EDTA addition. Corrosion 36:306-312

37. El Achouri M, Kertit S, Gouttaya HM, Nciri B, Bensouda Y, Perez L, Infante MR, Elkacemi K (2001) Corrosion inhibition of iron in $1 \mathrm{M} \mathrm{HCl}$ by some gemini surfactants in the series of 
alkanediyl $\alpha$ - $\omega$,-bis-(dimethyl tetradecyl ammonium bromide). Prog Org Coat 43:267-273

38. Macdonald JR, Johanson WB (1987) In: Macdonald JR (ed) Theory in impedance spectroscopy. Wiley, New York

39. Mertens SF, Xhoffer C, Decooman BC, Temmerman E (1997) Short-term deterioration of polymer-coated $55 \% \mathrm{Al}-\mathrm{Zn}$ - part 1: behavior of thin polymer films. Corrosion 53:381-388

40. Trabanelli G, Montecelli C, Grassi V, Frignani A (2005) Electrochemical study on inhibitors of rebar corrosion in carbonated concrete. J Cem Concr Res 35:1804-1813
41. Trowsdate AJ, Noble B, Haris SJ, Gibbins ISR, ThomsonG E, Wood GC (1996) The influence of silicon carbide reinforcement on the pitting behaviour of aluminium. Corros Sci 38:177-191

42. Reis FM, De Melo Costa HG (2006) Electrochemical impedance spectroscopy selection of papers from the 6th international symposium (EIS 2004). J Electrochim Acta 51:1375-1904 